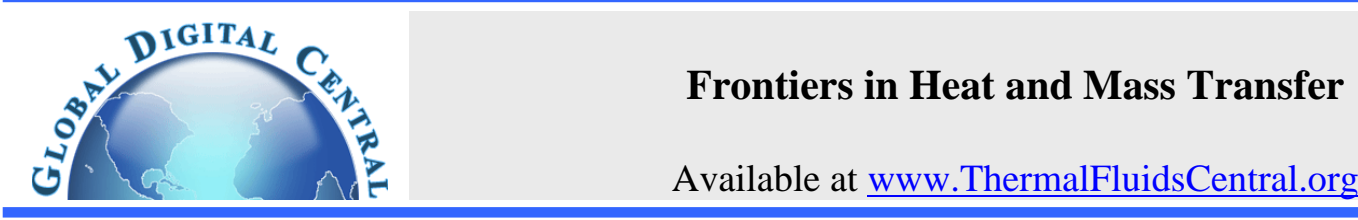

\title{
HEAT AND MASS TRANSFER FOR NATURAL CONVECTION MHD FLOW OVER A PERMEABLE MOVING VERTICAL PLATE WITH CONVECTIVE BOUNDARY CONDITION IN THE PRESENCE OF VISCOUS DISSIPATION
}

\author{
Stanford Shateyi \\ Department of Mathematics; School of Mathematical and Natural Sciences; University of Venda; \\ P. Bag X5050, Thoyandou; South Africa
}

\begin{abstract}
The spectral relaxation method is employed to examine natural convective heat and mass transfer, MHD flow over a permeable moving vertical plate with convective boundary conditions in the presence of viscous dissipation, thermal radiation and chemical reaction. The governing partial differential equations were transformed into a system of nonlinear ordinary differential equations by using a similarity approach. The resultant dimensionless ordinary equations were numerically solved by employing an effective Relaxation spectral algorithm with Chebyshev scheme. The pertinent results are then displayed in tabular form and graphically.

Keywords: Spectral relaxation method; convective heating; viscous dissipation; permeable plate
\end{abstract}

\section{INTRODUCTION}

Nonlinear partial differential equations are used to describe many real world phenomena. There is still a big challenging problem that is compelling scientists and engineers to seek different methods or to improve the existing numerical methods though great strides have been made in trying to obtain exact or approximate solutions of these nonlinear differential equations. In the current study, we wish to employ the recently developed method to numerically analyse the problem of heat and mass transfer for free convection MHD flow over a permeable moving vertical plate with convective boundary condition in the presence of viscous dissipation. The application of an external magnetic field has over the years to be a very important control mechanism in many industrial applications. MHD power generators, plasma studies, petroleum industries, cooling of nuclear reactors, the boundary layer control in aerodynamics and crystal growth are few important examples of magnetohydrodynamic flow of an electrically conducting fluid over a surface [Chen (2004) and (Damseh et al., 2006)] In areas where high temperatures are encountered, thermal radiation effect becomes very important. These areas include, high temperature plasmas, cooling of nuclear reactors, liquid metal fluids and power generation. Shateyi (2008) analysed thermal radiation and buoyancy effects on heat and mass transfer over a semi-infinite stretching surface. Machireddy (2014) studied the effects of Joule heating on MHD mixed convection boundary layer flow over a stretched vertical plate in the presence of thermal radiation and viscous dissipation. The process of converting mechanical energy of downward flowing water into thermal radiation and acoustical energy is referred viscous dissipation. Carefully designed devices in stream beds reduce the kinetic energy of flowing waters. This is meant to reduce their erosive potential on banks and river bottoms. (Abel et al., 2011) numerically analysed the problem of MHD flow and heat transfer of an incompressible viscous fluid with the presence of buoyancy force and viscous dissipation. The above researchers are restricted to either prescribed temperatures or heat flux at the wall or slip.

Aziz (2009)) introduced the idea of using convective boundary conditions which are the generalisation of isothermal and thermal slip boundary conditions. Magyari (2011) found an exact solution of the problem formulated by Aziz (2009). Ibrahim and Reddy (2013) examined steady laminar natural convection flow over a semi-infinite moving vertical plate with internal heat generation and convective surface boundary layer condition. Abdel-Rahman (2013) carried out an analysis of the problem of heat and mass transfer over a moving permeable flat stretching sheet in the presence of convective boundary layer conditions. (Rashidi et al., 2014) investigated combined heat and mass transfer by mixed convective flow along a moving flat plate with hydrodynamic slip and thermal convective boundary conditions.

The main goal of this paper is to find numerical solutions using a powerful technique namely, Spectral Relaxation Method (SRM) [(Motsa et al., 2013); Motsa and Makukula (2013)] for the velocity, temperature and concentration distributions to investigate the problem of heat and mass transfer free convection MHD flow over a permeable moving plate with convective boundary condition. We use a similarity transformation

${ }^{\dagger}$ Corresponding author. Email: stanford.shateyi@univen.ac.za 
approach to transform the governing partial differential equations into a set of nonlinear ordinary differential equations. The pertinent results are then displayed in tabular form and graphically. Approximations of Skin friction, the Nusselt number and Sherwood number which are very crucial from engineering applications point of view are also presented in the present study. It is hoped that the results obtained in this study will serve as a complement to previous studies and also provide useful information for further studies.

\section{MATHEMATICAL FORMULATION}

We investigate heat and mass transfer in a steady laminar two-dimensional boundary layer flow over a permeable moving plate. We assume that the plate is moving with a uniform velocity $U_{0}$ in contact with a quiescence cold fluid at temperature $T_{\infty}$ and concentration $C_{\infty}$. We also assume that the left side surface of the plate is heated by convection from a hot fluid at temperature $T_{f}$. This in turn provides a heat transfer coefficient represented by $h_{f}$. Under the Boussinesq and boundary-layer approximations, the boundary layer governing equations are as follows:

$$
\begin{aligned}
& \frac{\partial u}{\partial x}+\frac{\partial v}{\partial y}=0, \\
& u \frac{\partial u}{\partial x}+v \frac{\partial u}{\partial y}=\nu \frac{\partial^{2} u}{\partial y^{2}}-\frac{\sigma B^{2}}{\rho} u+g \beta_{T}\left(T-T_{\infty}\right) \\
& +g \beta_{C}\left(C-C_{\infty}\right), \\
& u \frac{\partial T}{\partial x}+v \frac{\partial T}{\partial y}=\alpha \frac{\partial^{2} T}{\partial y^{2}}+\frac{16 \sigma^{*} T_{\infty}^{3}}{3 \rho c_{p} k_{1}} \frac{\partial^{2} T}{\partial y^{2}}+\frac{\mu}{\rho c p}\left(\frac{\partial u}{\partial y}\right)^{2}, \\
& u \frac{\partial C}{\partial x}+v \frac{\partial C}{\partial y}=D \frac{\partial^{2} C}{\partial y^{2}}-k_{r}\left(C-C_{\infty}\right) .
\end{aligned}
$$
by:

The associated boundary conditions to the current model are given

$$
\begin{gathered}
u=U_{0}, v=V_{w},-\kappa \frac{\partial T}{\partial y}=h_{f}\left(T_{f}-T\right), C_{f}=C_{\infty}+b x \text { at } y=0 \\
u \longrightarrow 0, T \longrightarrow T_{\infty}, C \longrightarrow C_{\infty} \text { as } y \longrightarrow \infty
\end{gathered}
$$

\section{SIMILARITY ANALYSIS}

We introduce a similarity variable $\eta$, a dimensionless stream function $f(\eta)$, a dimensionless temperature $\theta(\eta)$, and a dimensionless concentration $\phi(\eta)$ in order to transform the governing partial differential equations into a system on nondimensional ordinary differential equations. The expressions for the dimensionless variables are as follows:

$$
\begin{aligned}
\eta=\sqrt{\frac{U_{0}}{\nu x}} y, u=U_{0} f^{\prime}, v=\frac{1}{2} \sqrt{\frac{U_{0}}{\nu x}}\left(\eta f^{\prime}-f\right), \theta(\eta) & =\frac{T-T_{\infty}}{T_{f}-T_{\infty}}, \\
\phi(\eta) & =\frac{C-C_{\infty}}{C_{f}-C_{\infty}} .
\end{aligned}
$$

The following system of ordinary equations is obtained upon substituting the similarity variables into equations (2) - (4).

$$
\begin{aligned}
& f^{\prime \prime \prime}+\frac{1}{2} f f^{\prime \prime}-M f^{\prime}+G r \theta+G c \phi=0, \\
& \left(\frac{4+3 R}{3 \operatorname{Pr} R}\right) \theta^{\prime \prime}+\frac{1}{2} f \theta^{\prime}+E c f^{\prime \prime 2}=0, \\
& \frac{1}{S c} \phi^{\prime \prime}+\frac{1}{2} f \phi^{\prime}-\gamma \phi=0 .
\end{aligned}
$$

The corresponding boundary conditions to the transformed equations are

$$
\begin{aligned}
& f(0)=f_{w}, f^{\prime}(0)=1, \quad \theta(0)=\frac{B_{i}}{1+B_{i}}, \phi(0)=1, \\
& f^{\prime}(\infty)=0, \quad \theta(\infty)=0, \phi(\infty)=0 .
\end{aligned}
$$

Where $f_{w}=-2 x^{1 / 2} V_{w} / U_{0}^{1 / 2} \nu^{1 / 2}$ is the suction/injection parameter $\left(f_{w}>0\right.$ for suction and $f_{w}<0$ for injection) and $B_{i}=h_{f} \sqrt{\nu x} / \kappa U_{0}$ is the Biot number, $M$ is the magnetic parameter, $G r$ is the Grashof number and $G c$ is the modified Grashof number, $\operatorname{Pr}$ is the Prandtl number, $R$ is the thermal radiation parameter, $E c$ is the Eckert number. The quantities of engineering interest in this study are the skin friction coefficient $C_{f}$ and the Nusselt number $N u_{x}$.

\section{METHODS OF SOLUTION}

We employ the Chebyshev pseudo-spectral method known as Spectral Relaxation Method (SRM) to solve the set of ordinary differential equations (8) - (10) together with the boundary conditions (11) and (12). This method transforms sets of nonlinear ordinary differential into sets of linear ordinary differential equations. We use a program written in MATLAB computer language to implemented the entire computational procedure. The fluid velocity, temperature, concentration, local skin friction coefficient, local Nusselt and Sherwood numbers are determined from these numerical computations. The SRM algorithm starts with the assumption of having a system of $m$ non-linear ordinary differential equations in $m$ unknowns functions, $z_{i}(\eta), i=1,2, \ldots ., m$ where $\eta \in[a, b]$ is the independent variable. To solve the resultant iterative scheme, we then use the Chebyshev pseudo-spectral method. The details of the spectral methods can be found in [(Canuto et al., 1988), Trefethen (2000)]. Before applying the spectral method, the domain on which the governing equation is defined is transformed to the interval $[-1,1]$ on which the spectral method can then be implemented. We use the transformation $\eta=(b-a)(\tau+1) / 2$ to map the interval [a,b] to $[-1,1]$.

To apply the SRM on the nonlinear ordinary differential equations, we first set $f^{\prime}(\eta)=g(\eta)$ and then write the equations as the following set of equations:

$$
\begin{aligned}
& f^{\prime}=g, \\
& g^{\prime \prime}+\frac{1}{2} f g^{\prime}-M g+G r \theta+G c \phi=0, \\
& \left(\frac{3+4 R}{P r}\right) \theta^{\prime \prime}+\frac{1}{2} f \theta^{\prime}+E c g^{2}=0, \\
& \phi^{\prime \prime}+\frac{1}{2} f \phi^{\prime}-\gamma \phi=0,
\end{aligned}
$$

and the boundary conditions become

$$
\begin{array}{r}
f(0)=f_{w}, g(0)=1, \theta^{\prime}(0)=-B_{i}(1-\theta(0)), \phi(0)=1, \\
g(\infty)=0, \theta(\infty)=0, \phi(\infty)=0 .
\end{array}
$$

In view of the SRM, we obtain the following iterative scheme:

$$
\begin{aligned}
& f_{r+1}^{\prime}=g_{r}, \quad f_{r+1}(0)=f_{w}, \\
& g_{r+1}^{\prime \prime}+\frac{1}{2} f_{r+1} g_{r+1}^{\prime}-M g_{r+1}=G r \theta_{r}-G c \phi_{r}, \\
& g_{r+1}(0)=1, \quad g_{r+1}(\infty)=0, \\
& \left(\frac{3+4 R}{P r}\right) \theta_{r+1}^{\prime \prime}+\frac{1}{2} f_{r+1} \theta_{r+1}^{\prime}=-E c g_{r+1}^{\prime 2}, \\
& \theta_{r+1}(0)=\frac{B_{i}}{1+B_{i}}, \theta_{r+1}(\infty)=0, \\
& \frac{1}{S c} \phi_{r+1}^{\prime \prime}+\frac{1}{2} f_{r+1} \phi_{r+1}^{\prime}-\gamma \phi_{r+1}=0 \\
& \phi_{r+1}(0)=1, \phi_{r+1}(\infty)=0 .
\end{aligned}
$$

The above equations form a system of linear decoupled equations which can be solved iteratively for $r=1,2, .$. , starting from initial guesses/approximations $\left(g_{0}(\eta), \theta_{0}(\eta), \phi_{0}(\eta)\right)$. 
Applying the Chebyshev pseudo-spectral method on (18) we obtain

$$
\begin{aligned}
& A_{1} \mathbf{f}_{r+1}=B_{1}, \quad f_{r+1}\left(\tau_{\bar{N}}\right)=f_{w} \\
& A_{2} \mathbf{g}_{r+1}=B_{2}, \quad g_{r+1}\left(\tau_{\bar{N}}\right)=1, \quad g_{r+1}\left(\tau_{0}\right)=0 \\
& A_{3} \theta_{r+1}=B_{3}, \quad \theta_{r+1}\left(\tau_{\bar{N}}\right)=\frac{B_{i}}{1+B_{i}}, \quad \theta_{r+1}\left(\tau_{0}\right)=0, \\
& A_{4} \phi_{r+1}=B_{4}, \quad \phi_{r+1}\left(\tau_{\bar{N}}\right)=1, \quad \phi_{r+1}\left(\tau_{0}\right)=0
\end{aligned}
$$

where

$$
\begin{aligned}
& A_{1}=\mathbf{D}, \quad B_{1}=\mathbf{g}_{\mathbf{r}}, \\
& A_{2}=\mathbf{D}^{2}+\operatorname{diag}\left[\frac{1}{2} \mathbf{f}_{i+1}\right] \mathbf{D}-M \mathbf{I}, \quad B_{2}=-G r \theta_{r}-G c \phi_{r}, \\
& A_{3}=\left(\frac{3+4 R}{P r}\right) \mathbf{D}^{2}+\operatorname{diag}\left[\frac{1}{2} \mathbf{f}_{i+1}, B_{3}=-E c g_{r+1}^{\prime 2},\right. \\
& A_{4}=\mathbf{D}^{2}+\operatorname{diag}\left[\frac{1}{2} \mathbf{f}_{i+1}\right] \mathbf{D}, B_{4}=0,
\end{aligned}
$$

where $I$ is the identity matrix of size $(\bar{N}+1) \times(\bar{N}+1), \mathbf{f}, \mathbf{g}, \phi$ and $\theta$ are the values of $f, g, \phi$ and $\theta$ respectively, when evaluated at the grid points. Equations (22) - (25) constitute the SRM scheme. The initial approximation required to start the iterative process is

$$
g_{0}(\eta)=f_{w}+1-e^{-\eta}, \theta_{0}(\eta)=\frac{B_{i}}{1+B_{i}} e^{-\eta}, \phi_{0}(\eta)=e^{-\eta} .
$$

which are randomly chosen functions that satisfy the boundary conditions. The iteration is repeated until convergence is achieved. The convergence of the SRM scheme is defined in terms of the infinity norm as

$E r=\operatorname{Max}\left(\left\|f_{r+1}-f_{r}\right\| ;\left\|g_{r+1}-g_{r}\right\| ;\left\|\theta_{r+1}-\theta_{r}\right\| ;\left\|\phi_{r+1}-\phi_{r}\right\|\right)$.

Accuracy of the scheme is established by increasing the number of collocation points $N$ until the solutions are consistent and further increases do not change the value of the solutions.

\section{RESULTS AND DISCUSSION}

We numerically solved the system of ordinary differential equations (8) to (10) subject to the boundary conditions (11) to (12) by using spectral relaxation method (SRM). This is a recently developed method and details of the method are found in Motsa et al (2013). The SRM results presented in this work were obtained using $N=50$ collocation points. The convergence was achieved after as few as five iterations. We take 30 to be the infinity value $\eta_{\infty}$. We use the following default values for the parameters, $\operatorname{Pr}=0.71, M=1, G r=G c=0.5, R=1, E c=$ $0.2, \gamma=0.1, f_{w}=1, B i=0.1, S c=0.22$.

Table 1 displays the results generated by SRM compared to those generated by the bvp $4 c$ method. When varying the suction/injection parameter $\left(f_{w}\right)$ for the skin friction coefficient. We see that the SRM results are exactly the same to those produced by the bvp $4 \mathrm{c}$ method. But we remark that the SRM converges much faster than the bvp $4 c$ method. We observe in Table 1 that the skin friction coefficient $\left|f^{\prime \prime}(0)\right|$ increases as fluid is injected into the flow system but decreases with suction. Table 2 displays the influence of the Biot number, magnetic parameter, thermal Grashof number, Eckert number as well as the chemical reaction parameter on the skin friction, Nusselt number and the Sherwood number. Increasing the values of the Biot number leads to the reduction of the skin friction but increases the rate of heat transfer but has little effect on the Sherwood number. We also observe in this table that the skin friction is greatly reduced with increasing values of the magnetic parameter as well as increasing values of the chemical reaction parameter. However, increasing values of the thermal Grashof number, as well as the Eckert number lead to reduced values of the skin friction. The Nusselt number is reduced by respectively, increasing values of the magnetic field parameter, thermal Grashof number, thermal radiation, chemical reaction as well

\begin{tabular}{|c|c|c|c|c|c|c|c|}
\hline$\overline{B_{i}}$ & $M$ & $G r$ & $E c$ & $\gamma$ & $-f^{\prime \prime}(0)$ & $-\theta(0)$ & $-\phi(0)$ \\
\hline$\overline{0.1}$ & & & & & 1.17648434 & 0.02296360 & 0.3624096 \\
\hline 0.2 & & & & & 1.14330592 & 0.05538486 & 0.3673157 \\
\hline 0.3 & & & & & 1.11538040 & 0.08302899 & 0.3683031 \\
\hline 0.4 & & & & & 1.09154929 & 0.10687331 & 0.3697321 \\
\hline & 0.5 & & & & 0.72776021 & 0.11615534 & 0.3780081 \\
\hline & 1 & & & & 1.09154928 & 0.10687331 & 0.3657582 \\
\hline & 3 & & & & 1.96846334 & 0.08393311 & 0.3428451 \\
\hline & 5 & & & & 2.52994777 & 0.07060280 & 0.3323940 \\
\hline & & & 0.0 & & 1.09348590 & 0.12042807 & 0.36566480 \\
\hline & & & 0.2 & & 1.08961835 & 0.09339418 & 0.36586812 \\
\hline & & & 0.4 & & 1.08577332 & 0.06666081 & 0.36608719 \\
\hline & & & 0.0 & & 1.11487871 & 0.06027164 & 0.36350618 \\
\hline & & & 0.5 & & 1.09524439 & 0.04769565 & 0.36562721 \\
\hline & & & 1.0 & & 1.07223341 & 0.03511602 & 0.36737067 \\
\hline & & & & 0 & 1.05912259 & 0.04465894 & 0.31903909 \\
\hline & & & & 1 & 1.17757295 & 0.02196190 & 0.62551055 \\
\hline & & & & 3 & 1.25379170 & 0.00832897 & 0.95182726 \\
\hline
\end{tabular}

as Eckert number. The Sherwood number is greatly reduced by increasing values of the magnetic parameter, but increases with increasing values of the chemical reaction parameter.

Table 1 Comparison of skin friction coefficient, Nusselt number the Sherwood number for different values of $f_{w}$.

\begin{tabular}{lll}
\hline \hline & $b v p 4 c$ & Present \\
\hline \hline$f_{w}$ & $-f^{\prime \prime}(0)$ & $-f^{\prime \prime}(0)$ \\
\hline-1 & 1.28286170 & 1.28286170 \\
-0.5 & 1.38287145 & 1.38287145 \\
0 & 1.49181813 & 1.49181813 \\
0.5 & 1.60996131 & 1.60996131 \\
\hline \hline
\end{tabular}

Table 2 Values of skin friction coefficient and Nusselt Number and the Sherwood number for different values of selected parameters

Figure 1 illustrates the influence of the magnetic field $M$ on the velocity distribution. We observe in this figure that increasing values of $M$ reduces the velocity profiles. This is because the application of a transverse magnetic field to an electrically conducting fluid physically gives rise to a resistive-type force called the Lorentz force. This force then slows down the motion of the fluid thereby delaying the transition of a laminar flow to a turbulent flow. Therefore an application of a controlled magnetic field can be used as a stabilising mechanism. We display the effect on the Biot number on the fluid velocity on Figure 2. Values if the Biot number greater than 0.1 imply that the heat convection away from the surface is much faster that the heat inside the body. This in turn gives rise to high temperature gradients. This physically explains why the velocity profiles increase with increasing values of the Biot number as can be clearly observed in Figure 2. Figure 3 shows the influence of suction/injection on the velocity profiles. Injecting/blowing $(f w<0)$ fluid into flow system causes the fluid velocity to increase. This in turn increases the velocity boundary layer thickness. This then accelerates the transition of a laminar boundary layer flow to a turbulent flow. However, opposite effects are experienced when suction is applied to the flow system. Figure 4 illustrates the influence of the thermal Grashof number on the velocity distribution. As expected the fluid velocity also increases as the values of the Grashof number increase.

Figure 5 displays the effect of the chemical reaction on the fluid velocity profiles. Velocity profiles are reduced as the values of the chemical reaction parameter increases. The influence of the Biot number on 


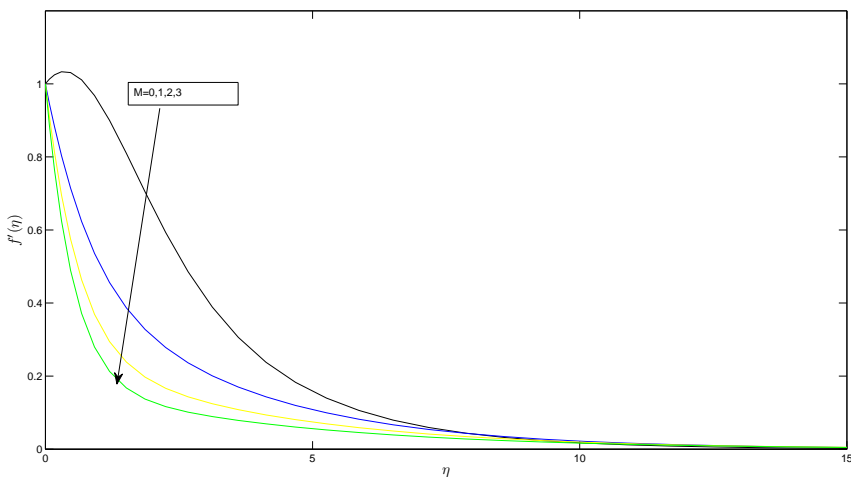

Fig. 1 The influence of the magnetic parameter on the dimensionless velocity profiles

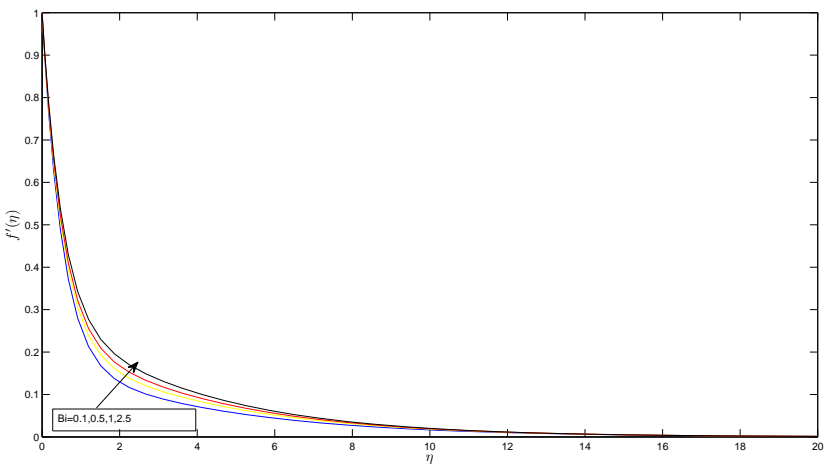

Fig. 2 The effect Biot number on the dimensionless velocity profiles

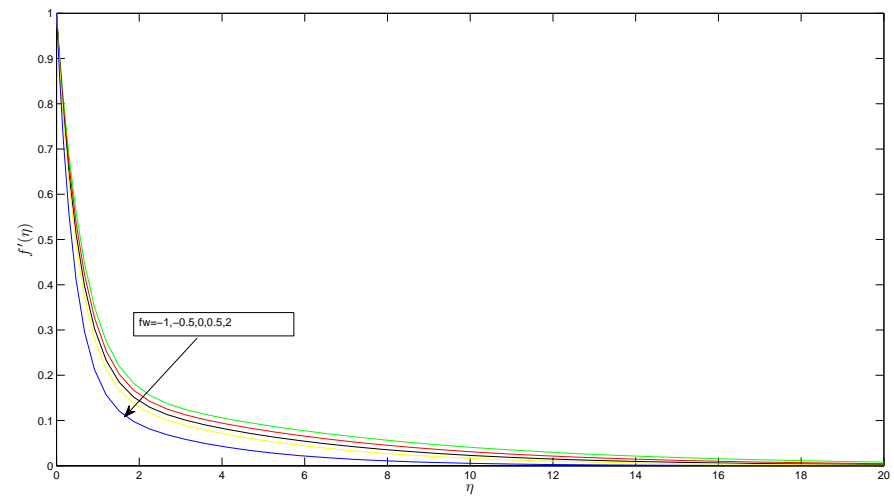

Fig. 3 The influence of the suction/injection parameter on the dimensionless velocity profiles

the temperature distribution is depicted on Figure 6. Mathematically, increasing the values of the Biot number implies that the convective heat transfer coefficient increases thereby enhancing more heat transfer from the surface. This then causes the fluid to heat up thus increasing the fluid temperature. Figure 7 displays the effect of the Eckert number on the temperature distribution, As expected we observe in Figure 7 that the temperature profiles greatly increase as the values of the Biot number increase. Physically, increasing the values of the Eckert number generates heat in the fluid due to frictional heating. Thus the effect of increasing $E c$ is to enhance the temperature at any point as can be clearly observed

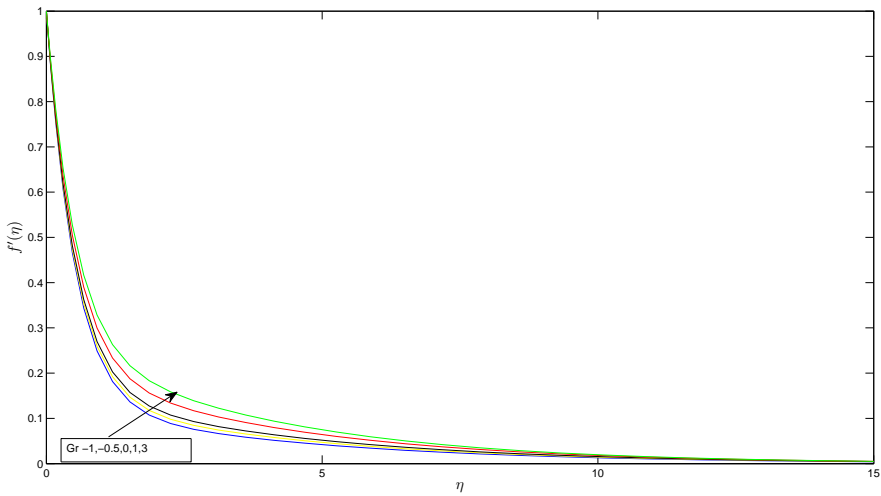

Fig. 4 The varation of the thermal buoyancy number on the dimensionless velocity profiles

in Figure 7. The influence the Prandtl number on the temperature distribution is depicted on Figure 8. We observe the effect of the Prandtl number on the temperature distribution. It can be clearly observed that the temperature as well as the thermal boundary layer rapidly decrease with increasing values of the Prandtl number.

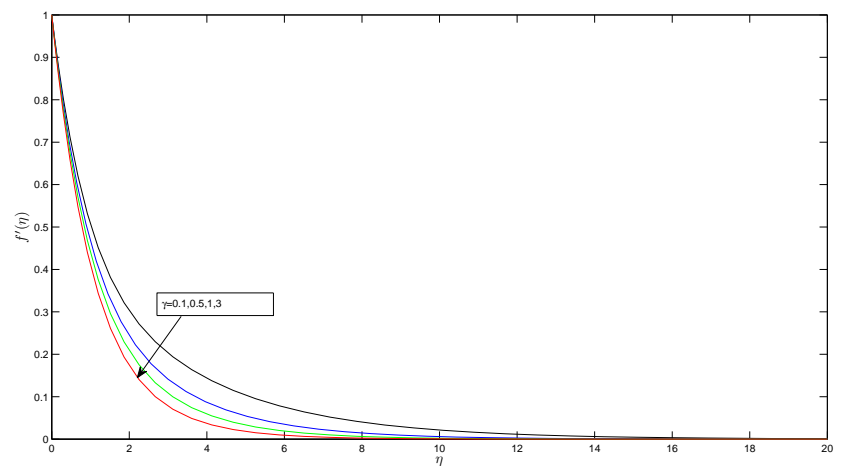

Fig. 5 The influence of the chemical reaction parameter on the dimensionless velocity profiles

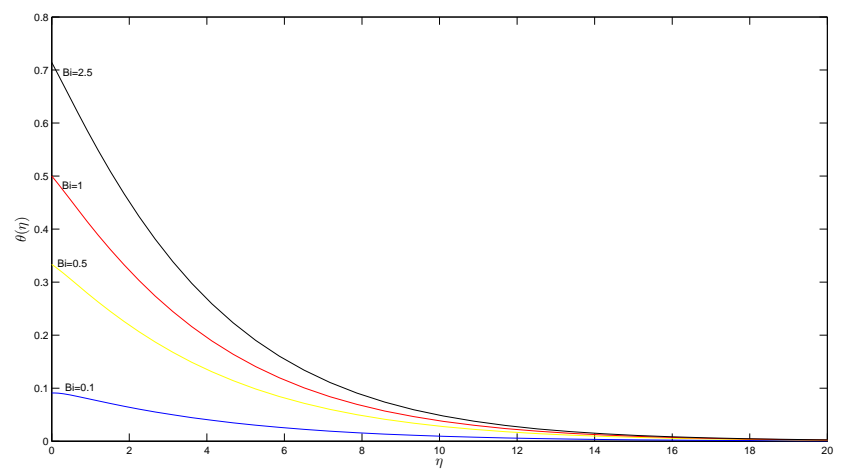

Fig. 6 The influence of the Biot number on the dimensionless temperature profiles

Figure 9 displays the effect of thermal radiation on the temperature distribution. It is observed from this figure that as the values of $R$ increase, the temperature profiles increase. Figure 10 shows the influence 


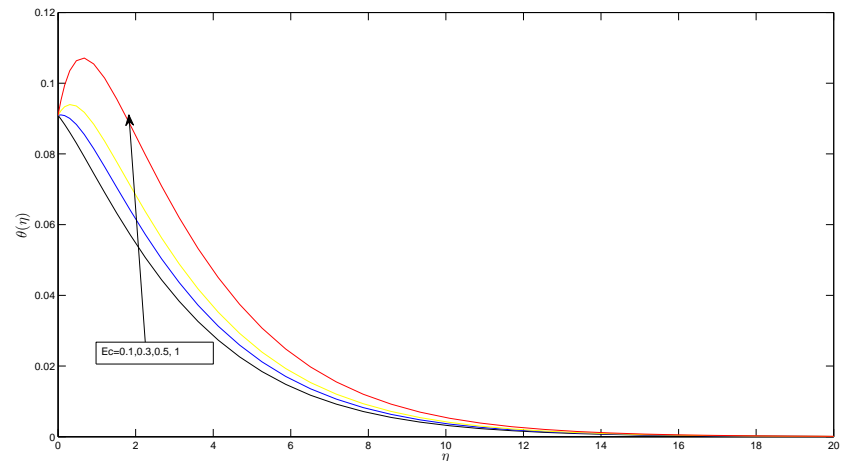

Fig. 7 The influence of the Eckert number on the dimensionless temperature profiles

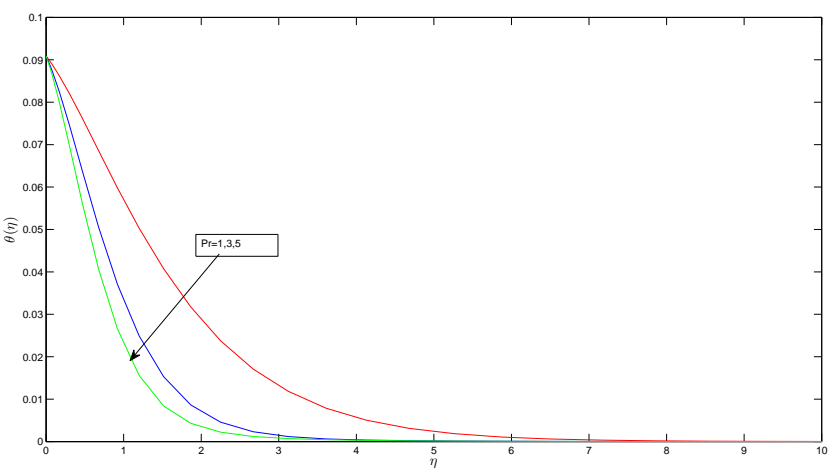

Fig. 8 Variation of the Prandtl number on the dimensionless velocity profiles

of suction/injection on the concentration profiles. We observe in Figure 10 that the concentration decreases as the suction parameter increases but increases as the injection parameter increases. It can be clearly seen that the concentration of the fluid decreases with increasing values of $\gamma$. The variation of the chemical reaction on the concentration profiles is depicted on 11. It can be clearly seen that the concentration of the fluid decreases with increasing values of $\gamma$. Lastly Figure 12 depicts the effect of the Schmidt number on the concentration profiles. We see from this Figure 12 that the concentration boundary layer thickness decreases as the Schmidt number $S c$ increases. This is because as $S c$ increases, the concentration becomes heavier.

\section{CONCLUSIONS}

We employed the spectral relaxation method to study the problem of heat and mass transfer for natural convection MHD flow over a permeable moving vertical plate with convective boundary condition in the presence of viscous dissipation, thermal radiation and a uniform chemical reaction. We transformed the governing partial differential equations into a system of nonlinear ordinary differential equations using a suitable similarity transformations. The resultant dimensionless ordinary equations were numerically solved by employing an effective Relaxation spectral algorithm with Chebyshev scheme. The velocity was found to increase with increasing values of the Biot number, buoyancy parameters, injection parameter and thermal radiation but decreases with increasing values of the magnetic parameter, suction, chemical reaction, Prandtl/Schmidt numbers. The fluid temperature increases with increasing values of the Biot number, Eckert number, radiation parameter and magnetic parame-

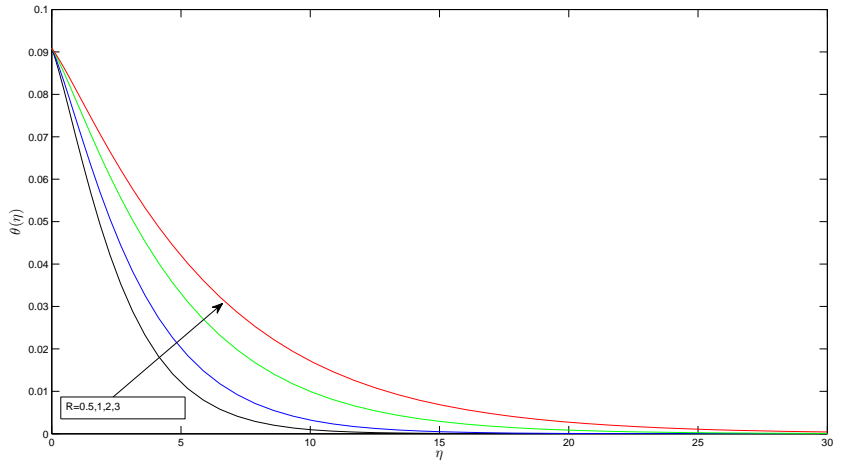

Fig. 9 The influence of the thermal radiation parameter on the dimensionless temperature profiles

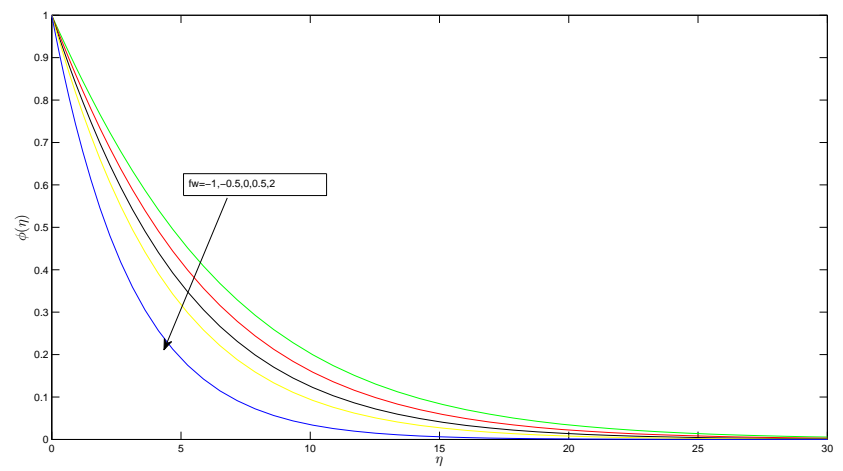

Fig. 10 Variation of suction/injection on the concentration profiles.

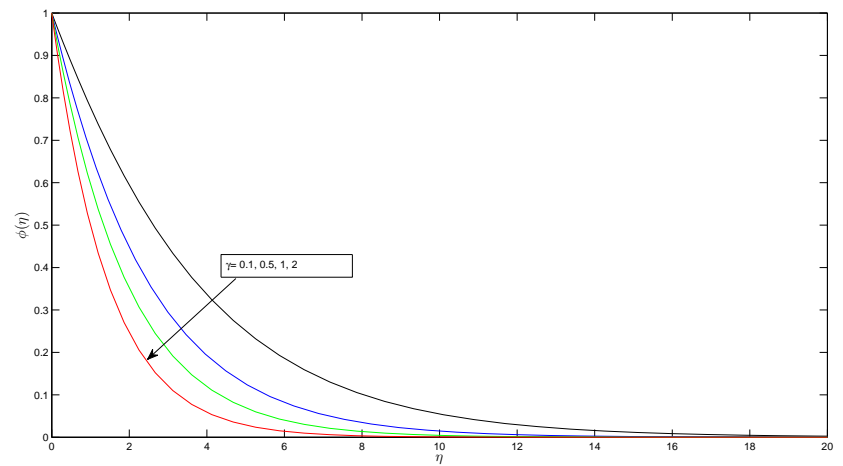

Fig. 11 Variation of chemical reaction parameter on the concentration profiles.

ter but decreases with increases values of the Prandtl number, buoyancy parameter and suction. Chemical reaction was found to greatly reduce the fluid concentration.

\section{ACKNOWLEDGEMENTS}

The author wishes to acknowledge financial support from the University of Venda and we are so grateful to the suggestions made by the reviewers. 


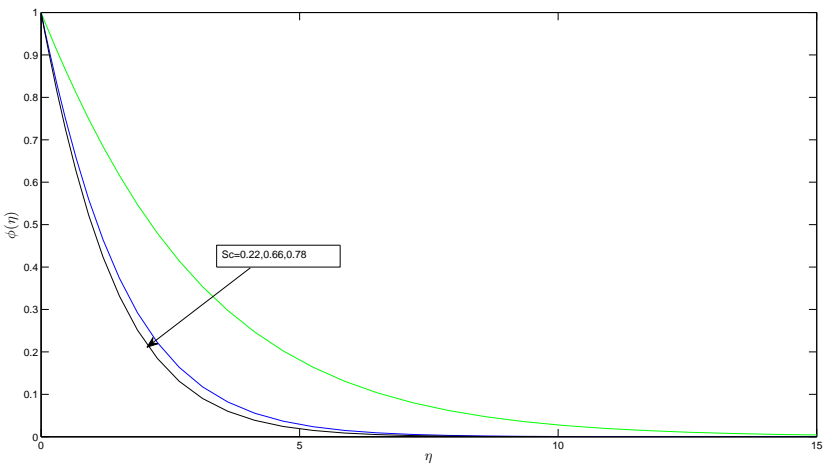

Fig. 12 Variation of Schmidt number on the concentration profiles

\section{NOMENCLATURE}

$B \quad$ magnetic field of constant strength

$B i \quad$ Biot number

$C \quad$ concentration

$C_{f} \quad$ fluid concentration

$c_{p} \quad$ heat capacity at constant pressure

$C_{\infty} \quad$ free concentration

$E c \quad$ Eckert number

$f(\eta) \quad$ dimensionless stream function

$g \quad$ acceleration due to gravity

Gr Grashof number

Gc modified grashof number

$h_{f} \quad$ heat transfer coefficient

$k_{r} \quad$ chemical reaction reaction parameter

$\mathrm{Nu} \quad$ local Nusselt number

$\mathrm{Pr} \quad$ Prandtl number

$(x, y) \quad$ cartesian coordinates along and normal to the plate

$(u, v) \quad$ velocity components along $x-$ and $y-$ axes

$U_{0} \quad$ plate velocity

$T \quad$ temperature

$T_{f} \quad$ the convective fluid temperature below the moving sheet

$T_{\infty} \quad$ free stream temperature

Greek Letters

$\alpha \quad$ thermal diffusivity of the porous medium

$\beta_{T} \quad$ volumetric thermal expansion coefficient

$\beta_{C} \quad$ volumetric solutal expansion coefficient

$\rho \quad$ fluid density

$\sigma \quad$ electrical conductivity

$\eta \quad$ similarity variable

$\mu \quad$ coefficient of viscosity

$\nu \quad$ kinematic viscosity

$\theta \quad$ dimensionless temperature

$\phi \quad$ dimensionless concentration

$\gamma \quad$ chemical reaction parameter

\section{REFERENCES}

Abdel-Rahman R.G., 2013, "MHD Slip Flow of Newtonian Fluid past a Stretching Sheet with Thermal Convective Boundary Condition, Radiation, and Chemical Reaction," Mathematical Problems in Engineering, Volume 2013, 1-12.

http://dx.doi.org/10.1155/2013/359817.
Abel M.S., Kumar, K.N., and Ravikumara., 2011, " MHD Flow, and Heat Transfer with Effects of Buoyancy, Viscous and Joules Dissipation over a Nonlinear Vertical Stretching Porous Sheet with Partial Slip," Engineering, 3, 285-291.

http://dx.doi.org/10.4236/eng.2011.33033.

Aziz A.,2009, "A Similarity Solution for Laminar Thermal Boundary Layer over Flat Plate with Convective Surface Boundary Condition," Commun Nonlin Sci Numer. Simul. 14(4), 1064-1068.

https://doi.org/10.1016/j.cnsns.2008.05.003.

Canuto, C., Hussaini, M.Y., Quarteroni, A., and Zang, T, A., 1988, Spectral Methods in Fluid Dynamics, Springer-Verlag, Berlin.

Chen, C.H., 2004, "Heat and Mass Transfer in MHD Flow by Natural Convection from a Permeable, Inclined Surface with Variable Wall Temperature and Concentration," Acta. Mech. 172(3-4), 219-235. http://dx.doi.org/10.1007/s00707-004-0155-5.

Damseh, R.A., Duwairi H.M., and Al-Odat, M., 2006, "Similarity Analysis of Magnetic Field and Thermal Radiation Effects on Forced Con-ection Flow," Turk. J. Eng. Env.Sci, 30, 83-89.

Ibrahim, S.M., and Reddy, N.B., 2013, "SIMILARITY Solution of Heat and Mass Transfer for Natural Convection over a Moving Vertical Plate with Internal Heat Generation and a Convective Boundary Condition in the Presence of Thermal Radiation, Viscous Dissipation, and Chemical Reaction," ISRN Thermodynamics, Volume 2013, 1-10 http://dx.doi.org/10.1155/2013/790604.

Machireddy, G.R., 2014, "Influence of Thermal Radiation, Viscous Dissipation and Hall Current on MHD Convection Flow over a Stretched Vertical Flat Plate," Ain Shams Engineering Journal, 5, 169-175. https://doi.org/10.1016/j.asej.2013.08.003.

Magyari, E., 2011, "COMMENT On a Similarity Solution for Laminar Thermal Boundary Layer over a Flat Plate with a Convective Surface Boundary Condition", Comm Nonlin Sci Numer Simul. 14, 1064-1068.

Motsa S.S., Dlamini P., and Khumalo M., 2013 , "A New Multistage Spectral Relaxation Method for Solving Chaotic Initial Value Systems," Nonlin-ear Dyn. 72(1-2), 265-283. http://dx.doi.org/10.1007/s11071-012-0712-8.

Motsa, S.S., and Makukula, Z., 2013, "ON Spectral Relaxation Method Approach for Steady von Kármán Flow of a Reiner-Rivlin Fluid with Joule Heating, Viscous Dissipation and Suction/Injection," Cent. Eur. J. Phys., 11(3)363-374 http://dx.doi.org/10.2478/s11534-013-0182-8.

Rashidi,M.M., Kavyani,N., Abelman,S., Uddin, M.J., and Freidoonimehr, N.,2014, "DOUBLE Diffusive Magnetohydrodynamic (MHD) Mixed Convective Slip Flow along a Radiating Moving Vertical Flat Plate with Convective Boundary Condition," PLoS ONE, 9(10): e109404. http://dx.doi.org/10.1371/journal.pone.0109404.

Shateyi, S., 2008 "THERMAL Radiation and Buoyancy Effects on Heat and Mass Transfer over a Semi-Infinite Stretching Surface with Suction and Blowing," Journal of Applied Mathematics, Volume 2008, 1-12. http://dx.doi.org/10.1155/2008/414830.

Trefethen, L. N. 2000, Spectral Methods in MATLAB, SIAM, Philadelphia. 